\title{
A fixed point theorem for multifunctions and an application
}

\author{
MARgReT HöFT
}

Abstract. The main result is a fixed point theorem for compositions of chain faithful multifunctions (Corollary 2.3). The theorem is then applied to get sufficient conditions for the fixed point property of the product of two partially ordered sets.

For the Special Issue on the NIH Conference on Universal Algebra \& Lattice Theory

\section{Introduction}

Let $P$ and $Q$ be partially ordered sets. A multifunction $F$ of $P$ into $Q$ is a function that assigns to an element $p \in P$ a non-empty subset $F(p)$ in $Q$. We use capital letters $F, G, H$ for multifunctions of $P$ into $Q$ and write $F: P \rightarrow Q$. Let $F: P \rightarrow Q$ and $G: Q \rightarrow R$ be two multifunctions, then the composition $G \circ F: P \rightarrow$ $R$ is defined as $(G \circ F)(p)=\cup\{G(q) \mid q \in F(p)\}$. A fixed point of a multifunction $F: P \rightarrow P$ is a point $p \in P$ such that $p \in F(p)$. If for all $p \in P, F(p)$ is a one-element subset of $Q$, then $F$ is a single-valued function in the usual sense, and a fixed point of $F$ is then a point $p=F(p)$, i.e. a fixed point in the usual sense. For single-valued functions we shall use $f, g, h$. A single-valued function $f: P \rightarrow Q$ is order preserving if $x_{1} \leqq x_{2}$ in $P$ implies $f\left(x_{1}\right) \leqq f\left(x_{2}\right)$ in $Q . P$ has the fixed point property (fpp) if every order preserving function $f: P \rightarrow P$ has a fixed point. The problem whether $P \times Q$ has the fpp if both $P$ and $Q$ have the $f p p$ is an unsolved problem at present. For current information on the fixed point problem one may consult [4]. In [6] and in [8] multifunctions have been used to get results about the fpp of a product $P \times Q$. In section 2 we prove a fixed point theorem for compositions of multifunctions (Theorem 2.2) and use it in section 3 to obtain fixed points for order preserving maps on products $P \times Q$ (Theorem 3.1, Theorem 3.3, Theorem 3.6).

Presented by R. P. Dilworth. Received January 12, 1987. Accepted for publication in final form April 27, 1987. 
The following lemma will be used in section 3. It appears in various forms in the literature, e.g. [4, Theorem, p. 288], [2, Theorem, p. 402] and is a special case of a more general result on the $f p p$ of sets that are bounds of chains, e.g. [6, Lemma 1, p. 62].

LEMMA 1.1. If $P$ has the fpp and $C$ is a chain in $P$, then the sets $U=\{u \mid u \geqq x$ for all $x \in C\}$ and $V=\{v \mid v \leqq x$ for all $x \in C\}$ have the fpp, in particular, both $U$ and $V$ are non-empty.

Note, that if $C=\{p\}$ is a one-element chain, $U=\llbracket p)$ and $V=(p\rfloor$, the principal upper and lower ends generated by $p$, have the fpp.

\section{A fixed point theorem for multifunctions}

We introduce several conditions that a multifunction $F: P \rightarrow Q$ may satisfy:

(1) If $x_{1} \leqq x_{2}$ in $P$ and if $y_{1} \in F\left(x_{1}\right)$, then there is $y_{2} \in F\left(x_{2}\right)$ such that $y_{1} \leqq y_{2}$ in $Q$.

(2) If $x_{1} \leqq x_{2}$ in $P$ and if $y_{2} \in F\left(x_{2}\right)$, then there is $y_{1} \in F\left(x_{1}\right)$ such that $y_{1} \leqq y_{2}$ in $Q$.

In [5] a multifunction satisfying (1) and (2) is called an order preserving multifunction, and we shall adopt this terminology here too. Note, that if $F$ is single-valued, then $F$ satisfies (1) and (2) if and only if $F$ is an order preserving function in the usual sense. Also, if $G: Q \rightarrow R$ is another order preserving multifunction, then the composition $G \circ F: P \rightarrow R$ will be order preserving.

Now let $C$ be a chain in $P$, and suppose there is an order preserving function $f: C \rightarrow Q$ such that $f(x) \in F(x)$ for all $x \in C$.

(3) If $x_{0}=\sup C$, then there exists $y_{0} \in F\left(x_{0}\right)$ such that $f(x) \leqq y_{10}$ for all $x \in C$.

(4) If $x_{0} \geqq x$ for all $x \in C$, then there exists $y_{0} \in F\left(x_{0}\right)$ such that $f(x) \leqq y_{0}$ for all $x \in C$.

The dual conditions of (3) and (4) will be needed too, they will be referred to as conditions (3d) and (4d). A multifunction that satisfies (4) or (4d), will obviously also satisfy (1), (3) or (2), (3d) respectively. If $P$ is chain finite, conditions (3) and (3d) are trivially fulfilled and (4) and (4d) reduce to (1) and (2).

If $P$ has the property that the least upper bounds (greatest lower bounds) exist for all non-empty chains in $P$, we say that $P$ has suprema (infima) of chains. If $P$ has both suprema and infima of chains, we say that $P$ is chain-complete. 
LEMMA 2.1. Suppose $P$ has suprema of chains and let $F: P \rightarrow Q$ be a multifunction. Then $F$ satisfies (4) if and only if $F$ satisfies (1) and (3).

Proof. Let $F$ satisfy (1) and (3), let $C$ be a non-empty chain in $P$, let $f: C \rightarrow Q$ be such that $f(x) \in F(x)$ for all $x \in C$, let $x_{0}=\sup C$ and let $x_{1} \geqq x$ for all $x \in C$. By (3) there is $y_{0} \in F\left(x_{0}\right)$ so that $f(x) \leqq y_{0}$ for all $x \in C$. Now $x_{0} \leqq x_{1}$ and by (1) there is $y_{1} \in F\left(x_{1}\right)$ so that $y_{0} \leqq y_{1}$. Also $f(x) \leqq y_{0} \leqq y_{1}$ for all $x \in C$, and (4) is verified.

THEOREM 2.2. Suppose $P$ has suprema of chains, let $F: P \rightarrow Q$ and $G: Q \rightarrow P$ be multifunctions that satisfy condition (4). If there are elements $p$, $r \in P, q \in Q$ so that $q \in F(p), r \in G(q)$ and $p \leqq r$, then $G \circ F$ has a fixed point $p_{0} \geqq p$ in $P$.

Proof. Let $\mathscr{L}$ be a system of chains of triples $(x, y, z)$ where $x, y \in P, z \in Q$, $z \in F(x), y \in G(z)$ and $x \leqq y$ in $P$ and which contain the triple $(p, r, q)$ as least element. $\mathscr{L}$ is non-empty, since by assumption $\{(p, r, q)\}=: C \in \mathscr{L}$. Also, the union of a chain of such chains in $\mathscr{L}$ is again in $\mathscr{L}$, therefore Zorn's Lemma can be applied, and there is a maximal chain $C_{0}$ in $\mathscr{L}$.

Let $X=\left\{x \mid(x, y, z) \in C_{0}\right\}, \quad Y=\left\{y \mid(x, y, z) \in C_{0}\right\}, \quad Z=\{z \mid(x, y, z) \in$ $\left.C_{0}\right\} . X, Y, Z$ are chains and by assumption on $P, x_{0}=\sup X$ exists in $P$ and $x_{0} \geqq p$. We define $f: X \rightarrow Q$ as $f(x)=z \in F(x)$ and $g: Z \rightarrow P$ as $g(z)=y \in G(z)$ if and only if $(x, y, z) \in C_{0}$. Since $F$ satisfies condition (3), there is $z_{0} \in F\left(x_{0}\right)$ such that $f(x)=z \leqq z_{0}$ for all $x \in X$, hence all $z \in Z$. $G$ satisfies condition (4), therefore, for $z_{0} \geqq z$ for all $z \in Z$, there is $y_{0} \in G\left(z_{0}\right)$ so that $g(z)=y \leqq y_{0}$ for all $z \in Z$ and hence all $y \in Y$. Now $x \leqq y \leqq y_{0}$ for all $(x, y, z)$ in $C_{0}$, thus $x_{0} \leqq$ $y_{0} \in G\left(z_{0}\right)$. For the triple $\left(x_{0}, y_{0}, z_{0}\right)$ we have now $(x, y, z) \leqq\left(x_{0}, y_{0}, z_{0}\right)$ for all $(x, y, z) \in C_{0}$ and $z_{0} \in F\left(x_{0}\right), y_{0} \in G\left(z_{0}\right), x_{0} \leqq y_{0}$. Since $C_{0}$ is a maximal chain, it must be true, that $\left(x_{0}, y_{0}, z_{0}\right) \in C_{0}$, and $\left(x_{0}, y_{0}, z_{0}\right)$ is the largest element of $C_{0}$.

We prove now that $x_{0} \geqq p$ is a fixed point of $G \circ F$. Let $x_{1}:=y_{0} \in G\left(z_{0}\right)$, then $x_{0} \leqq x_{1}$ and $z_{0} \in F\left(x_{0}\right)$. Since $F$ satisfies condition (1), there is $z_{1} \in F\left(x_{1}\right)$, so that $z_{0} \leqq z_{1}$. Since $y_{0} \in G\left(z_{0}\right)$ and since $G$ satisfies condition (1), there is $y_{1} \in G\left(z_{1}\right)$ so that $y_{0} \leqq y_{1}$. Now $\left(x_{0}, y_{0}, z_{0}\right) \leqq\left(x_{1}, y_{1}, z_{1}\right), z_{1} \in F\left(x_{1}\right), y_{1} \in G\left(z_{1}\right)$ and $x_{1} \leqq y_{1}$, hence $\left(x_{1}, y_{1}, z_{1}\right) \in C_{0}$ and therefore $\left(x_{0}, y_{0}, z_{0}\right)=\left(x_{1}, y_{1}, z_{1}\right)$. Moreover, $x_{0}=x_{1} \in$ $G\left(z_{0}\right), z_{0} \in F\left(x_{0}\right)$, i.e. $x_{0} \in \cup\left\{G(u) \mid u \in F\left(x_{0}\right)\right\}$, and $x_{0}$ is a fixed point of $G \circ F$.

In Theorem 2.2 we can replace condition (4) for $F$ and $G$ by (4d) and get the dual result.

A multifunction satisfying (4) and (4d) will be called chain faithful. Note, that chain faithful multifunctions are order preserving, and the composition of two chain faithful multifunctions is therefore order preserving, but in general not chain faithful. 
COROLLARY 2.3. Suppose $P$ is chain complete, let $F: P \rightarrow Q$ and $G: Q \rightarrow P$ be chain faithful multi-functions. If there are comparable elements $p, r$ in $P$ and $q \in Q$, so that $q \in F(p)$ and $r \in G(q)$, then $G \circ F$ has a fixed point $p_{0}$ comparabie to $p$ in $P$.

If $Q$ in Corollary 2.3 has an element $x_{0} \in Q$ that is comparable to any other element of $Q$, then we can take $p \in G\left(x_{0}\right)$, and for any $q \in F(p)$ we have $x_{0} \leqq q$ or $q \leqq x_{0}$. Since $G$ is order preserving, there is $r \in G(q)$ so that $p \leqq r$ or $r \leqq p$ and $q \in F(p)$.

COROLLARY 2.4. Let $P, Q, F, G$ be as in Corollary 2.3. If $Q$ has an element $x_{0}$ comparable to any other element of $Q$, then $G \circ F$ has a fixed point.

Let us now take $P=Q$ and let $G: P \rightarrow P$ be the single-valued identity function $G(p)=\{p\}$ for all $p \in P$. $G$ then trivially satisfies condition (4), and the requirement in Theorem 2.2 , that there are elements $p \leqq r$ in $P, q \in Q$, where $q \in F(p)$ and $r \in G(q)$, simplifies to the assumption that there are elements $p \leqq r=q \in F(p)$. Theorem 1.1 in [7] is now an immediate consequence of Theorem 2.2 above:

COROLLARY 2.5 (R. E. Smithson). Suppose that $P$ has suprema of chains, and let $F: P \rightarrow P$ be a multifunction satisfying (1) and (3). If there is a point $p \in P$. and a point $r \in F(p)$ so that $p \leqq r$, then $F$ has a fixed point.

The proof of this fact in [7, Theorem 1.1] appears to be incorrect. The same idea that was used in [7] to prove Theorem 1.1 was used again to prove Theorem 1.9 in [7]. In the latter case not only the proof but the theorem itself turn out to be incorrect, a counterexample is given in [3].

An order preserving single valued function $f: P \rightarrow P$ is chain faithful, and in this case, the self dual version of Corollary 2.5 is the well known result, that $f$ has a fixed point, if there is an element $p$ comparable to $f(p)$, e.g. [1, Theorem 2].

\section{Applications to the fixed point property for products}

To apply Theorem 2.2 and its corollaries to the fixed point property for products, we use the notation and terminology of [6]: Let $P, Q$ be partially ordered sets and let $h: P \times Q \rightarrow P \times Q$ be an order preserving function. Then $h(p, q)=(g(p, q), f(p, q))$ for all $p \in P$ and $q \in Q$, where $g: P \times Q \rightarrow P$ and $f: P \times Q \rightarrow Q$ are order preserving functions. We define multifunctions $F: P \rightarrow Q$ 
and $G: Q \rightarrow P$ as $F(p)=\{q \mid f(p, q)=q\}$ and $G(q)=\{p \mid g(p, q)=p\}$. Clearly, $F(p)$ and $G(q)$ are non-empty sets, if both $P$ and $Q$ have the fixed point property for single-valued functions.

Here is the connection to Theorem 2.2:

THEOREM 3.1. Suppose $P$ and $Q$ have the fpp. Then $h: P \times Q \rightarrow P \times Q$ has a fixed point if and only if $G \circ F$ has a fixed point.

Proof. $(p, q)=h(p, q)=(g(p, q), f(p, q))$, if and only if $p \in G(q)$ and $q \in F(p)$, if and only if $p \in(G \circ F)(p)$.

Theorem 2.2 can only be applied to multifunctions satisfying condition (4) of section 2. The multifunctions $F$ and $G$ defined above are of this type:

THEOREM 3.2. Suppose $P$ and $Q$ have the fpp. Then $F$ and $G$ are chain faithful.

Proof. We show that $F$ satisfies condition (4). All the other cases are similar.

Let $C$ be a chain in $P$, let $x_{0} \geqq x$ for all $x \in C$, let $h: C \rightarrow Q$ be order preserving so that $h(x) \in F(x)$, i.e. $f(x, h(x))=h(x)$. Note, that $x_{1}$ exists because of Lemma 1.1. Let $U=\{y \mid y \geqq h(x)$ for all $x \in C\} \subset Q$. By Lemma 1.1, $U \neq \emptyset$, and for $y \in U$ we have $f\left(x_{0}, y\right) \geqq f\left(x_{0}, h(x)\right) \geqq f(x, h(x))=h(x)$ for all $x \in C$, i.e. $f\left(x_{0}, y\right) \in U . U$ has the fpp by Lemma 1.1, therefore, there is $y_{0} \in U$ so that $f\left(x_{0}, y_{0}\right)=y_{0}$, hence $y_{01} \in F\left(x_{0}\right)$ and $y_{0} \geqq h(x)$ for all $x \in C$. $F$ therefore satisfies condition (4).

A multifunction $H: P \rightarrow P$ is a composition function if it is representable as $H=G \circ F$ where $F: P \rightarrow Q$ and $G: Q \rightarrow P$ are chain faithful multifunctions. Composition functions are order preserving multifunctions, every order preserving single-valued function is a composition function, and if $P$ has the property that every composition function has a fixed point, then $P$ has the $f p p$.

THEOREM 3.3. Let $P$ have the property that every composition function on $P$ has a fixed point. For every $Q$ with the $f p p, P \times Q$ has the $f p p$.

Proof. Let $h: P \times Q \rightarrow P \times Q$ be order preserving and let $F, G$ be as defined before. $H=G \circ F$ is a composition function by Theorem 3.2 and has a fixed point $p \in H(p)$ by assumption. By Theorem 3.1, $h$ has a fixed point.

Theorem 3.3 extends Theorem 1 of [6]. As a consequence of Theorem 3.2 and Corollary 2.3 we have

THEOREM 3.4. Suppose $P$ and $Q$ have the $f p p, P$ is chain complete. The 
following conditions are equivalent.

(1) $h: P \times Q \rightarrow P \times Q$ has a fixed point.

(2) $G \circ F$ has a fixed point.

(3) There are elements $p, r \in P, q \in Q$ so that $q \in F(p), r \in G(q)$ and $p \leqq r$ or $r \leqq p$.

(4) There are elements $p, r \in P, q \in Q$ so that $f(p, q)=q, g(r, q)=r$ and $r \leqq p$ or $p \leqq r$.

As we have seen in Corollary 2.4 the conditions in Theorem 3.4 are trivially satisfied if $Q$ has an element that is comparable to every other element of $Q$ :

COROLLARY 3.5. Suppose $P$ and $Q$ have the fpp, $P$ is chain complete, $Q$ has an element comparable to every other element in $Q$. Then $P \times Q$ has the fpp.

In combination with Lemma 1.1, Corollary 3.5 implies for instance, that if $P$ and $Q$ have the $f p p$ and $P$ is chain complete, then for all $p \in P, q \in Q, P \times[q)$, $P \times(q],[p) \times(q],(p] \times(q]$ all have the $f p p$.

Finally we derive an extension of Theorem 2 of [6] from Corollary 2.5 and Theorem 3.4, if we let $H=G \circ F$, with $F$ and $G$ as above.

THEOREM 3.6. Let $P$ be chain complete such that for every composition function $H$ there is a comparable pair $p, r \in P, r \in H(p)$. For every $Q$ with the fpp, $P \times Q$ has the fpp.

\section{REFERENCES}

[1] S. ABIAN and A. B. BRown, A theorem on partially ordered sets, with applications to fixed point thearems, Canad. J. Math. 13 (1961), 78-82.

[2] T. S. Fofanova, On the fixed point property of partially ordered sets, Coll. Math. Soc. Janos Bolyai, 33. Contributions to Lattice Theory, Szeged (Hungary) 1980, 401-406.

[3] H. HöfT, Order preserving selections for multifunctions, Contributions to General Algebra 5.

[4] I. Rival, The problem of fixed points in ordered sets, Ann. Discrete Math. 8(1980), 283-292.

[5] I. Rival, The fixed point property, Order 2 (1985), 219-221.

[6] A. RUTKowsKI, Multifunctions and the fixed point property for products of ordered sets, Order 2 (1985), 61-67.

[7] R. E. SMithson, Fixed points of order preserving multifunctions, Proc. A.M.S. Vol. 28 (1971), 304-310.

[8] J. W. WALKER, Isotone relations and the fixed point property for posets, Discrete Math. 48 (1984), $275-288$. 